\title{
Second-order wave loads on offshore structures using the Weber's transform method
}

\author{
M. Rahman ${ }^{1} \&$ S. H. Mousavizadegan ${ }^{2}$ \\ ${ }^{1}$ Department of Engineering Mathematics, Dalhousie University, Canada \\ ${ }^{2}$ Department of Mechanical Engineering, Dalhousie University, Canada
}

\begin{abstract}
The second-order wave loads are computed for the diffraction of monochromatic waves by a surface-piercing vertical cylinder in the finite and infinite fluid depths. The Weber transform method which is essentially a Hankel transform method with a more general kernel, is applied to compute the second-order force due to the second-order velocity potential. Suitable closed contours in the complex plane are used to derive the analytical solution of the improper integrals involved in this study. This makes the present solution distinct from the other available solution of the second-order forces.
\end{abstract}

\section{Introduction}

The fluid viscosity and the irrotational flow are important in determining the wave induced loads on offshore structures. The wave loading estimations for small volume structures are based on the well-known "Morison Equation" which involves both viscous drag and inertia forces. If the characteristic dimension of the structure is comparable to the wavelength, the diffraction theory should be applied to find the wave induced loads upon the structure.

There are some fundamental second-order phenomena that can not be predicted in the linearized wave theory. The second-order phenomena in monochromatic waves are the steady mean drift forces and the oscillating forces with a frequency twice the first order frequency. The force oscillating with the difference of frequencies that cause slow-drift motion of moored structures and the loads with sum frequencies that cause springing on TLPs are the second-order phenomena in multi-chromatic waves. 
Hunt and Baddour [2] derived a second-order solution for the diffraction of a nonlinear progressive wave in fluid of infinite depth, incident on a vertical, surfacepiercing, circular cylinder. They applied a modified form of Weber's integral theorem to obtain the second-order diffracted velocity potential and the associated wave force. Newman [5] analyzed the second-order wave force on a vertical cylinder by the application of the Weber transformation to derive the second-order potential. Solutions for the second-order forces associated with the first and the second-order velocity potentials are evaluated directly from pressure integration over the cylinder surface for the case of infinite fluid depth. He extended the solution to the case of finite fluid depth.

Rahman [6] extended the Lighthill's [3] second-order theory to the cases of intermediate and shallow fluid depth waves. Buldakov et al. [1] studied the diffraction problem of a unidirectional incident wave group by a bottom-seated cylinder. The amplitude of the incoming wave was assumed to be small in comparison with other linear scales of the problem to develop the corresponding second-order perturbation theory. They used the Fourier transform to treat time variation and separated spatial variables in solving the non-homogeneous second-order problem. The Weber transform is adopted to find the solution of the second-order velocity potential and the associated wave force. The computations are carried out in fluid of finite and infinite depths.

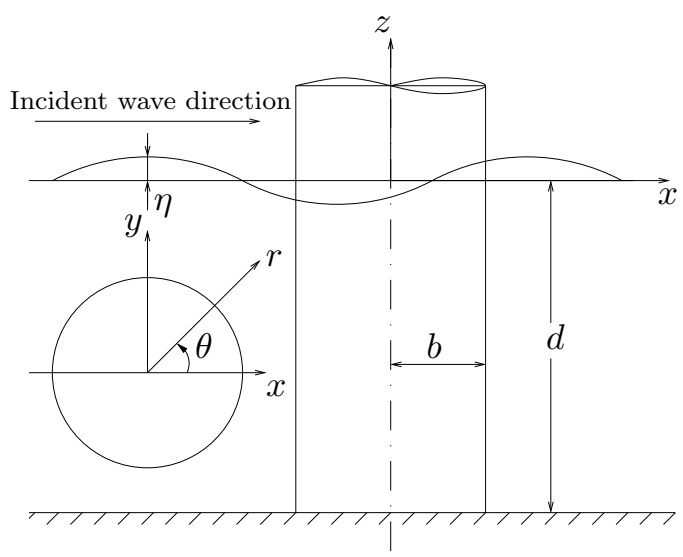

Figure 1: Schematic diagram of the circular surface piercing cylinder of radius $b$.

\section{Governing mathematical equations}

A rigid vertical cylinder of radius $b$ is acted upon by a train of regular progressive surface waves of amplitude $A$ (Fig.1). It is assumed that the fluid is incompressible, inviscid and the motion is irrotational. The fluid flow field can be defined by a scalar function called velocity potential and denoted by $\Phi(r, \theta, z, t)$. If the analysis 
is approximated up to the second-order, it can be written that $\Phi=\Phi_{\ell}+\Phi_{q}$, where $\Phi_{\ell}$ and $\Phi_{q}$ are the linear and the quadratic diffraction velocity potentials. The motion of the fluid is subjected to the Laplace equation in fluid domains, a free-surface kinematic boundary condition and a free-surface dynamic boundary condition. The fluid flow field is also subjected to a bottom condition that indicates no flux of mass through the bottom of the fluid, a radiation condition at a large distance from the body and a body surface boundary condition.

The total horizontal force acting upon the surface of the cylinder is obtained by the integration of the pressure along the surface of the cylinder. The fluid pressure is determined using Bernoulli's equation. The second-order force is partly due to the contribution of the first-order potential and partly due to the effect of the second-order potential. Using Weber's transform the contribution of the secondorder potential is derived and computed by direct integration of the attributed pressure along the surface of the cylinder.

\section{The second-order velocity potential}

This section is devoted to obtain the explicit expression of the second-order potential with the help of Weber's transform for the infinite and finite depth ocean. The mathematical developments are given below for each case.

\subsection{Infinite fluid depth}

The second-order velocity potential may be written in the form of $\Phi_{q}=\phi_{q} e^{-i 2 \omega t}$. The time independent quadratic potential $\phi_{q}$ can be expressed by the Fourier series in the form of

$$
\phi_{q}(r, \theta, z)=\sum_{n=0}^{\infty} \phi_{q}^{(n)}(r, z) \cos n \theta
$$

where the Fourier coefficients $\phi_{q}^{(n)}(r, z)=\frac{\alpha_{n}}{2 \pi} \int_{0}^{2 \pi} \phi_{q}(r, \theta, z) \cos n \theta d \theta$ in which $\alpha_{0}=1$ and $\alpha_{n}=2$ for $n \geq 1$.

The modified Weber transform that is an extension of the Hankel's transform with a more general kernel is applied to find the solution of the second-order velocity potential. If the term $\hat{\phi}(k)$ is denoted as the transformation of $\phi(r)$, the transform pairs are

$$
\begin{gathered}
\hat{\phi}^{(n)}(k)=\int_{b}^{\infty} \phi^{(n)}(r) W_{n}(k b, k r) r d r \\
\phi^{(n)}(r)=\int_{0}^{\infty} \hat{\phi}^{(n)}(k) \frac{W_{n}(k b, k r)}{{J_{n}^{\prime}}^{2}(k b)+Y_{n}^{\prime 2}(k b)} k d k,
\end{gathered}
$$

where $W_{n}(k b, k r)=J_{n}(k r) Y_{n}^{\prime}(k b)-Y_{n}(k r) J_{n}^{\prime}(k b)$ is the kernel for the integral transformation. 
Substituting (1) in the Laplace equation in cylindrical coordinates and taking the Weber transform of it, an ordinary differential equation is obtained,

$$
\hat{\phi}_{z z}^{(n)}(k, z)-k^{2} \hat{\phi}^{(n)}(k, z)=0
$$

The solution $\hat{\phi}^{(n)}(k, z)=\hat{\phi}^{(n)}(k) e^{k z}$ satisfies (3). Using the transformation (2), a solution for the $\theta$-independent quadratic velocity potential is constructed in the form

$$
\phi_{q}^{(n)}(r, z)=\int_{0}^{\infty} \hat{\phi}_{q}^{(n)}(k) e^{k z} W_{n}(k b, k r) \frac{k d k}{{J_{n}^{\prime}}^{2}(k b)+Y_{n}^{\prime 2}(k b)}
$$

This solution satisfies the body surface boundary and bottom boundary conditions. The multiplication of the solution (4) by $\cos n \theta$ also satisfies the Laplace equation. The solution (4) satisfies the free surface boundary condition provided that

$$
\hat{\phi}^{(n)}(k)=\frac{2 \omega \nu A^{2} \alpha_{n}}{k-4 \nu} \hat{S}^{(n)}(k),
$$

where $\hat{S}^{(n)}(k)$ is the transformation of $S^{(n)}(\nu r)$. The function $S^{(n)}(\nu r)$ is defined by

$$
S^{(n)}(\nu r)=i^{n+1} \sum_{m=0}^{\infty}(-1)^{m} B_{m n}(\nu r)+\frac{i^{n+1}}{2} \sum_{p=1}^{n-1} C_{p n}(\nu r)
$$

where

$$
\begin{aligned}
B_{m n}(\nu r)=A_{m}(\nu r) A_{m+n}(\nu r)+A_{m}^{\prime}(\nu r) A_{m+n}^{\prime}(\nu r) & + \\
& \frac{m(m+n)}{\nu^{2} r^{2}} A_{m}(\nu r) A_{m+n}(\nu r) \\
C_{p n}(\nu r)= & A_{p}(\nu r) A_{n-p}(\nu r)+A_{p}^{\prime}(\nu r) A_{n-p}^{\prime}(\nu r)-\frac{p(n-p)}{\nu^{2} r^{2}} A_{p}(\nu r) A_{n-p}(\nu r) .
\end{aligned}
$$

The time-independent quadratic velocity potential may be expressed in the form

$$
\begin{array}{r}
\phi_{q}(r, \theta, z)=2 \omega \nu A^{2} \sum_{n=0}^{\infty} \alpha_{n} \cos n \theta \int_{0}^{\infty} \frac{\hat{S}^{(n)}(k)}{k-4 \nu} e^{k z} W_{n}(k b, k r) \times \\
\frac{k d k}{J_{n}^{\prime 2}(k b)+Y_{n}^{\prime 2}(k b)}
\end{array}
$$

that satisfies the governing equations and all the boundary conditions. 


\subsection{Finite fluid depth}

The second-order time-independent velocity potential may be expressed for the case of finite fluid depth as

$$
\begin{gathered}
\phi_{q}(r, \theta, z)=\frac{2 g K^{2} A^{2}}{\omega} \sum_{n=0}^{\infty} \alpha_{n} \cos n \theta \int_{0}^{\infty} \hat{T}^{(n)}(k) \frac{\cosh k(z+d)}{k \sinh k d-4 \nu \cosh k d} \\
\frac{W_{n}(k b, k r)}{J_{n}^{\prime 2}(k b)+Y_{n}^{\prime 2}(k b)} k d k,
\end{gathered}
$$

where $\hat{T}^{(n)}(k)$ is the Weber transform of $T^{(n)}(K r)$ and

$$
T^{(n)}(K r)=S^{(n)}(K r)+\frac{3}{2} i^{n+1} \operatorname{sech}^{2} K d E^{(n)}(K r) .
$$

The function $S^{(n)}(K r)$ is expressed in (6) and $E^{(n)}(K r)$ is the extra term due to the limitation of the fluid depth,

$$
\begin{gathered}
E^{(n)}(K r)=\sum_{m=0}^{\infty}(-1)^{m}\left[\lambda_{m} H_{m}^{(1)}(K r) A_{m+n}(K r)+\lambda_{m+n} H_{m+n}^{(1)}(K r) \times\right. \\
\left.J_{m}(K r)\right]+\sum_{p=1}^{n-1}\left[\lambda_{p} H_{p}^{(1)}(K r) A_{n-p}(K r)+\lambda_{n-p} H_{n-p}^{(1)}(K r) J_{p}(K r)\right] .
\end{gathered}
$$

The complete derivation of the velocity potentials in infinite and finite fluid depth can be found in Mousavizadegan [4].

\section{The quadratic force}

This section contains the evaluation of second-order forces for the infinite and finite depth ocean. The analytical solutions are described below.

\subsection{Infinite fluid depth}

The quadratic force may be expressed in the form

$$
F_{q}=\Re\left\{f_{q} e^{-i 2 \omega t}\right\},
$$

where $\Re$ stands for the real part. The contribution of the solution $\phi_{q}(r, \theta, z)$ to the time-independent quadratic force stems only from the term $n=1$ in (7). The non-dimensional time independent quadratic force $\hat{f}_{q}=\frac{f_{q}}{\rho g A^{2} b}$ can be obtained by 
integrating the transient second-order pressure along the surface of the cylinder. Carrying out the integration, it can be written that

$$
\hat{f}_{q}=-\frac{4 i \nu}{b} \int_{b}^{\infty} G(\nu r) S^{(1)}(\nu r) r d r
$$

where

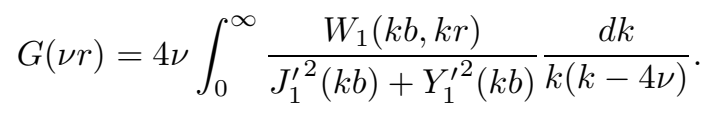

The integral in (12) is evaluated by the contour integration in the complex $k$-plane. The suitable contour for this problem is the semi-circular contour of infinite radius on the right side of the imaginary axis which contains the first and fourth quadrants. There exists one singularity $k=4 \nu$ which lies on the path of integration along the real axis.

Finally, the quadratic force can be obtained from,

$$
\begin{gathered}
\hat{f}_{q}=\frac{i d_{0}(\nu b)}{\nu b}\left\{\int_{0}^{\infty} \frac{K_{0}(4 \nu b y)}{y^{2}\left(1+y^{2}\right) K_{1}^{\prime}(4 \nu b y)} d y-\frac{\pi}{2}\left[\frac{H_{0}^{(2)}(4 \nu b)}{H_{1}^{\prime(2)}(4 \nu b)}+\frac{H_{0}^{(1)}(4 \nu b)}{H_{1}^{\prime(1)}(4 \nu b)}\right]\right\} \\
+\frac{4 i}{b} \int_{b}^{\infty}\left\{\int_{0}^{\infty} \frac{K_{1}(4 \nu r y)}{y\left(1+y^{2}\right) K_{1}^{\prime}(4 \nu b y)} d y-\frac{\pi}{2}\left[\frac{H_{1}^{(2)}(4 \nu r)}{H_{1}^{\prime(2)}(4 \nu b)}+\frac{H_{1}^{(1)}(4 \nu r)}{H_{1}^{\prime(1)}(4 \nu b)}\right]\right\} Z(\nu r) d r,
\end{gathered}
$$

where

$$
\begin{gathered}
d_{0}(\nu b)=\frac{2 i}{\pi} \sum_{m=0}^{\infty}(-1)^{m} \epsilon_{m} \lambda_{m}, \quad Z(\nu r)=\sum_{m=1}^{\infty} d_{m}(\nu b) H_{m}^{(1)}(\nu r) A_{m}(\nu r) \\
d_{m}(\nu b)=(-1)^{m+1} m\left(\lambda_{m+1}-\lambda_{m-1}\right) \quad m \geq 1, \quad \lambda_{m}=\frac{J_{m}^{\prime}(\nu b)}{H_{m}^{\prime(1)}(\nu b)}
\end{gathered}
$$

This result is similar to the one obtained by Newman [5]. The difference is in the solution of the contour integral for $G(\nu r)$. Here, the solution is a combination of the first and second kind of Hankel's functions. In contrast, Newman's result is expressed only by the second kind of the Hankel function.

\subsection{Finite fluid depth}

Because of orthogonal properties of the cosine functions, only the term proportional to $\cos \theta$ in (8) contributes to the second-order force due to the second-order potential. The second-order force coefficient can be obtained for the case of finite fluid depth by

$$
\hat{f}_{q}=-\frac{4 i K}{b} \int_{b}^{\infty} G(K r) T^{(1)}(K r) r d r,
$$


where

$$
\begin{gathered}
G(K r)=\frac{2 K}{i} \int_{0}^{\infty}\left\{\frac{H_{1}^{(2)}(k r)}{H_{1}^{(2)^{\prime}}(k b)}-\frac{H_{1}^{(1)}(k r)}{H_{1}^{(1)^{\prime}}(k b)}\right\} \frac{k^{-1} \sinh k d d k}{k \sinh k d-4 \nu \cosh k d} \\
=\frac{2 K}{i}\left(I_{2}-I_{1}\right)
\end{gathered}
$$

The integral $I_{1}$ can be computed by using the contour integration along a semicircular contour above the real axis. The integral $I_{2}$ is computed using a semicircular contour below the real axis. The integrand contains a singular point along the real axis and infinite number of singular points along the imaginary axis.

The final solution for the quadratic force coefficient is found for the case of finite fluid depth as

$$
\begin{aligned}
& \hat{f}_{q}=\frac{4 i d_{0}(K b)}{b}\left\{\sum_{n=1}^{\infty} \frac{g_{n} K_{0}\left(\kappa_{n} b\right)}{\kappa_{n} K_{1}^{\prime}\left(\kappa_{n} b\right)}-\frac{g_{0}}{\kappa_{0}}\left[\frac{H_{0}^{(1)}\left(\kappa_{0} b\right)}{H_{1}^{\prime(1)}\left(\kappa_{0} b\right)}+\frac{H_{0}^{(2)}\left(\kappa_{0} b\right)}{H_{1}^{\prime(2)}\left(\kappa_{0} b\right)}\right]\right\} \\
&+\frac{4 i}{b} \int_{b}^{\infty}\left\{\sum_{n=1}^{\infty} g_{n} \frac{K_{1}\left(\kappa_{n} r\right)}{K^{\prime}{ }_{1}\left(\kappa_{n} b\right)}-g_{0} \int_{b}^{\infty}\left[\frac{H_{1}^{(1)}\left(\kappa_{0} r\right)}{H_{1}^{(1)}\left(\kappa_{0} b\right)}+\frac{H_{1}^{(2)}\left(\kappa_{0} r\right)}{H_{1}^{(2)}\left(\kappa_{0} b\right)}\right]\right\} Z(K r) d r \\
&+\frac{6 i K}{b} \operatorname{sech}^{2} K d \int_{b}^{\infty}\left\{\sum_{n=1}^{\infty} g_{n} \frac{K_{1}\left(\kappa_{n} r\right)}{K^{\prime}{ }_{1}\left(\kappa_{n} b\right)}\right. \\
&\left.-g_{0} \int_{b}^{\infty}\left[\frac{H_{1}^{(1)}\left(\kappa_{0} r\right)}{H_{1}^{\prime(1)}\left(\kappa_{0} b\right)}+\frac{H_{1}^{(2)}\left(\kappa_{0} r\right)}{H_{1}^{\prime(2)}\left(\kappa_{0} b\right)}\right] E^{(1)}(K r) r d r\right\}
\end{aligned}
$$

where coefficients $g_{0}$ and $g_{n}$ are defined by

$$
g_{0}=2 \pi K\left\{\frac{4 \nu / \kappa_{0}}{\kappa_{0}^{2} d+4 \nu-16 \nu^{2} d}\right\}, \quad g_{n}=4 \pi K\left\{\frac{4 \nu / \kappa_{n}}{\kappa_{n}^{2} d-4 \nu+16 \nu^{2} d}\right\} .
$$

The terms denoted by $\kappa_{0}$ and $\kappa_{n}, n=1,2,3 \ldots$ are the roots of the transcendental equations $\kappa_{0} \tanh \kappa_{0} d=4 \nu$ and $\kappa_{n} \tan \kappa_{n} d=-4 \nu$, respectively. The first and the second part of (16) are the same as the force equation (13) for the infinite fluid depth case. The last part is an extra term due to the limitation of the fluid depth.

\section{Results and discussion}

The solutions contain considerable interactions of the Bessel and the modified Bessel functions of various kinds and orders. These functions are evaluated with double-precision routines. All computations are carried out using a PC with an Intel(R) Pentium(R) $4 \mathrm{CPU} 1.80 \mathrm{Ghz}$ and total memory of $384 M B$.

The second-order force coefficient $\hat{f}_{q}$ is obtained through the solution of (13) for the case of infinite fluid depth. The force equation (13) consists of four parts. The calculation of the second part is straightforward. There are three infinite integrals. The integrand of the first consists of a combination of the modified Bessel function of second kind. The second integral is a double infinite integral. The integrand of this integral consists of a combination of the modified Bessel function of the 
second kind of order one and an infinite series. This series consists of the Bessel and Hankel functions of the first kind of different orders. The integrand of the third infinite integral consists of a combination of the Hankel function of the first and the second kinds. It also consists of the infinite series as explained already. These integrals are calculated by the Simpson three-eights rule.

The computation of the first integral is straightforward. The solution for each $\nu b=$ const. is carried out in two steps. First the infinite interval is divided into small segments of $2^{k}-2^{k-1}, k=0,1,2, \cdots$ and $k-1 \geq 0$. The result on each subinterval is obtained for a convergence error in order of $10^{-6}$. The solutions for the segments are added together to reach an error less than $10^{-8}$. The second integral is a double infinite integral. The computation are carried out for each step ( $\nu b=$ const.), while $r$ is varied from $b$ to infinity. The result for the infinite series is obtained with an error less than $10^{-8}$. The result of the infinite internal integral is obtained in the same way as mentioned in the last paragraph. The third integral is also found by the Simpson method of three-eights rule with an error less than $10^{-6}$. However, the integrand of this integral has a very oscillatory nature. The computations are very time-consuming for large values of $\nu r$.

The solutions for the real and imaginary parts of the second-order force coefficients $\hat{f}_{q}$ are displayed in Fig. 2. They are compared with the published results of Newman [5]. The imaginary part of both solutions has the same sign and almost the same value. The real part of solutions has a different sign due to the different direction of the incoming waves. The values of the real part for small values of the $\nu b$ are also different. The differences are due to the different contours that are used in the integration process. It seems that our computations are more reliable due to the fact that the function $G(\nu r)$ in (10) is a real function. Newman's solution [5] for (10) is a complex function, while ours is a real one.

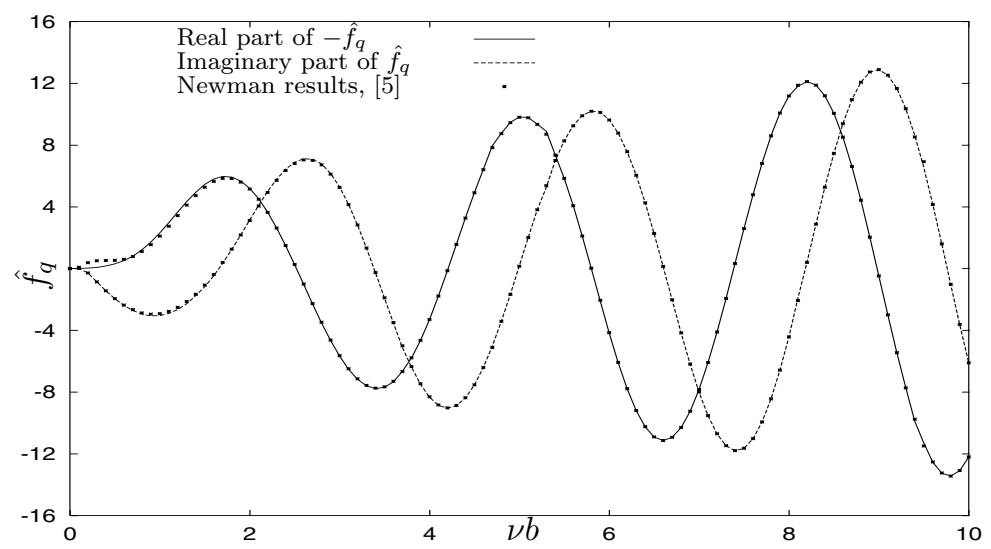

Figure 2: Real and imaginary parts of the non-dimensional quadratic force in infinite fluid depth. 
The quadratic force coefficient in finite depth is obtained through the solution of (16) for different depth-radius ratios. The first and second parts in (16) are the same as (10) in the case of infinite fluid depth. However, the infinite integrals from zero to infinity are replaced by an infinite series that has very good convergence properties and makes the computations faster. The third part is an extra term due to the limitation of the fluid depth.
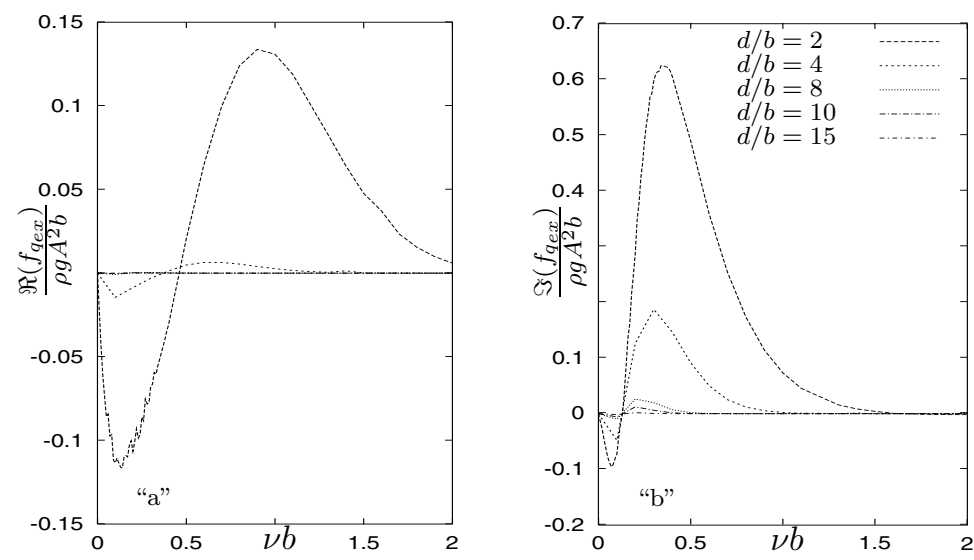

Figure 3: Real and imaginary parts of the extra term due to the limitation of the fluid depth.

The extra part is denoted by $\hat{f}_{q_{e x}}$. This part consists of two infinite integrals. The computation of the first integral is quite fast due to the proper behavior of the modified Bessel function. The second integral is very oscillatory and converges slowly. This part of the computation is the most time-consuming part. The real and imaginary parts of $\hat{f}_{q_{e x}}$ are depicted in Fig. 3. The contribution of this part is relatively small to the quadratic force. The quadratic force coefficient $\hat{f}_{q}$ is shown in Fig. 4. The infinite integral was solved using the Simpson three-eights rule with an error less than $5 \times 10^{-6}$. The infinite series converged with an error of order $10^{-8}$ or less. The effect of the limitation of depth is obvious and diminishes with an increase in the depth to radius ratio. This part of the wave force is affected by the limitation of fluid depth in a wide range of frequency spectrum.

\section{Conclusions}

Using the Weber transform, the second-order diffraction potential is evaluated in both cases of infinite and finite fluid depths. The quadratic force coefficients, due to the effect of the second-order velocity potential, are obtained using the direct integration of the related transient pressure around the cylinder surface. The resulting force coefficient is an integral along a horizontal distance from the vicinity of the 


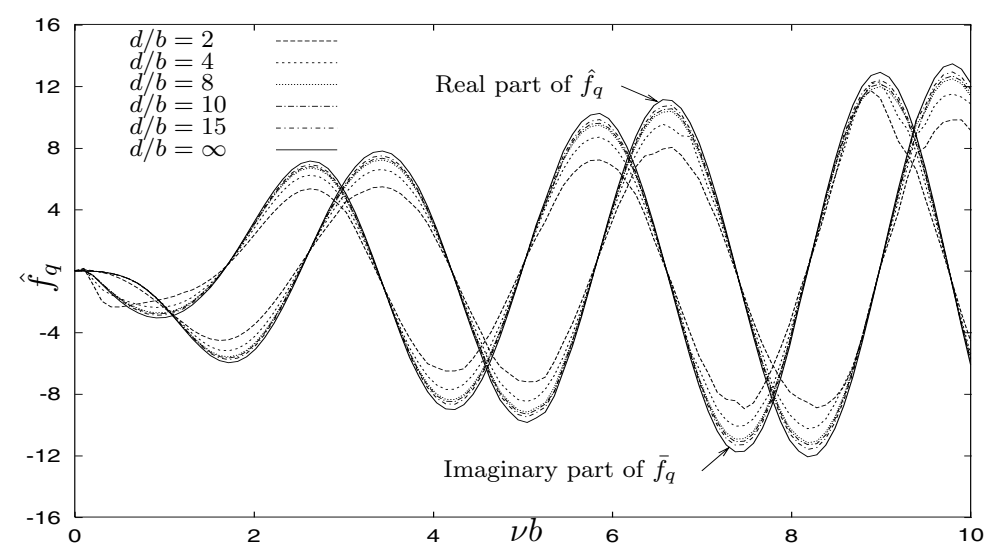

Figure 4: Real and imaginary parts of the quadratic force in various depth to radius ratios.

cylinder to infinity. The integrand of this integral contains an improper integral from zero to infinity of a real function. The expected solution is also a real function for the internal integral. The contour integration rule is adopted to obtain the solution of the internal integrals. The solutions are real functions for both infinite and finite fluid depth.

\section{Acknowledgement}

The Natural Sciences and Engineering Research Council (NSERC) of Canada is gratefully acknowledged.

\section{References}

[1] E. V. Buldakov, R. Eatock-Taylor, and P. H. Taylor, Local and far-field surface elevation around a vertical cylinder in unidirectional steep wave groups, Ocean Engineering, Vol. 31, pp. 833-864, 2004.

[2] J. N. Hunt and R. E. Baddour, The diffraction of nonlinear progressive waves by a vertical cylinder, Quarterly Journal of Mechanics and Applied Mathematics, Vol. 34, pp. 69-88, 1981.

[3] M. J. Lighthill, Waves and hydrodynamic loading, Proc. 2nd int. conf. on behavior of offshore structures, Vol. 1, pp. 1-40, 1979.

[4] S. H. Mousavizadegan, Analytical and numerical approaches to determine the second-order forces in wave-body interactions, Ph.D. thesis, Dalhousie University, Sept. 2005.

[5] J. N. Newman, The second-order wave force on a vertical cylinder, J. Fluid Mech., Vol. 320, pp. 417-443, 1996.

[6] M. Rahman, Nonlinear hydrodynamic loading on offshore structures, Theoret. Comput. Fluid Dynamics, Vol. 10, pp. 323-347, 1998. 